OBSERVATIONS

OX THE

\title{
BLOOD-CORPUSCLES AND PUS-GLOBULES
}

\author{
IN CERTAIN ANIMALS.
}

By GEORGE GULLIVER, Esq., F.R.S., F.Z.S.,

ASSISTANT SURGEON TO THE ROYAL REGIMENT OF HORSE GUARDS.

Communicated by JOHN G. PERRY, Esq.

READ NOVEMBER $26 \mathrm{TH}, 1839$.

Since the time of John Hunter, it has been supposed that the globules of pus are merely the red particles of the blood, deprived of their colouring matter, and modified in form and size by the inflammatory process. At an early period of my inquiries I was led to entertain this hypothesis, as I believe had been previously done by the late Dr. Young, Sir Astley Cooper, Mr. Mayo, and Dr. Carswell, as well as many other eminent pathologists. On the continent, indeed, M. Gendrin asserted that he had actually seen, by means of the microscope, the bloodcorpuscles transformed into those of pus. But Professor Müller and Dr. Güterbock did not assent to the supposition that the globules of pus were only the red particles of the blood which had undergone 
a change; and some observations on the elementary structure of the pus-globule, which I communicated to the society last spring, led me to believe that there was an essential difference between the corpuscles of blood and those of pus, notwithstanding the many remarkable circumstances in favour of the old opinion. The variety, for instance, in the magnitude of the blood corpuscles, is very great, and the disproportion in size and form between them and the globules of pus almost wholly disappears, if the former be subjected, during examination, to the action of certain reagents.

During last year at Windsor, with the assistance of Mr. Siddall, I made a series of experiments on the blood and pus of various animals. But my observations, though very numerous, did not afford such satisfactory results as might have been anticipated; for the size of the globules of pus is so extremely variable, that however small the corpuscles of the blood were in the animal under examination, there would certainly be some globules of pus equally small, while others of greater magnitude might possibly have been formed by the aggregation of the more minute granules. It might be supposed that the average size of the pus globules would be the same in one species, but this did not appear to be the case: thus in the human subject, pus from an abscess in the neck presented globules, of which the most common size was $\frac{1}{2666}$ th of an inch; the extreme diameters being $\frac{1}{4000}$ th and $\frac{1}{1333}$ rd, and numerous molecules were seen from $\frac{1}{12000}$ th to 
$\frac{1}{8000}$ th of an inch in diameter. In a case of gonorrhoea examined at the same time, the common size of the globules, which were remarkably definite and regular, was $\frac{1}{3000}$ th of an inch; this was on the fourth day of the disease. The globules examined again, on the ninth day, were most frequently $\frac{1}{2400}$ th of an inch in diameter, the extreme sizes being $\frac{1}{3200}$ th and $\frac{1}{2000}$ th; on the 12th day the average sized globules were again $\frac{1}{3000}$ th of an inch. The variation of their magnitude was not less remarkable in many brutes. In the horse, the most frequent size of the globules of some pus from an abscess in the groin was $\frac{1}{3000}$ th; from an abscess in the same horse's leg, a few days subsequently, the particles were generally smaller, the most frequent diameter being $\frac{1}{3200}$ th, with numerous globules of $\frac{1}{4000}$ th and $\frac{1}{5333}$ rd of an inch. The corpuscles of the blood in this animal are uniformly smaller than in man, so that a partial view might lead to the conclusion that this experiment was valuable in establishing the relation between the blood corpuscle and that of pus. But it would be tedious to relate how many contradictory results I obtained by reference merely to what appeared to be the average magnitude of particles so variable in this respect as the globules of pus.

Hence it became necessary to extend the inquiry to animals possessing oval blood-corpuscles. As I have related elsewhere, ${ }^{*}$ experiments on reptiles and

* Lond. and Edin. Phil. Mag., Sept. 1838, and Medical Gazette, Nov. 1st, 1839. 
birds were unsatisfactory. The recent discovery by M.Mandl, of the elliptical form of the blood disks of the dromedary and paco, led me to examine them in the vicugna and lama, in both of which I observ-: ed that the particles of the blood also possessed an extremely well-defined oval figure. The remarkable difference between these and the disks hitherto known in any other mammal, is a very interesting fact in the comparative anatomy of the blood. The following notes of these corpuscles were made at the time of the examination. The measurements are given in fractions of an English inch; the first indicates the small, and the last above the line the large extreme; the intervening numbers were obtained from disks which were present in abundance.

1. Dromedary (Camelus dromedarius).

Long diameter,

$1-4266$

$1-4000$

$1-3200$

1- -3000

$1-2460$

Average . . $\overline{1-3254}$

Short diameter,

$1-7110$

$1-6600$

$1-6400$

$1-5333$

$1-4800$

Average . . $\overline{1-5921}$. 
The disks were very flat in relation to the diameters, the edges being only $\frac{1}{20000}$ th to $\frac{1}{15000}$ th of an inch thick; their surfaces presented no projection whatever, and no nucleus could be detected in them by the most careful manipulation, with the aid of various reagents.

2. Paco (Auchenia paco). The disks scarcely differed in form and size from those of the dromedary.

3. Guanaco or wild lama (Auchenia glama). The corpuscles also as in the dromedary.

4. Vicugna (Auchenia vicugna). The corpuscles slightly smaller than in the preceding animals.

$$
\begin{gathered}
\text { Long diameter, } \\
1-5333 \\
1-4000 \\
1-3555 \\
1-3200 \\
1-2666
\end{gathered}
$$

Average . . 1-3555

$$
\begin{array}{cc} 
& \text { Short diameter, } \\
& 1-8000 \\
& 1-7110 \\
& 1-6400 \\
& 1-6000 \\
& 1-5333 \\
\text { Average } \quad . \quad & 1-6444
\end{array}
$$


The shortest corpuscles are often the broadest; these appearing nearly circular, and occasionally perfectly so. It was interesting to observe in the blood of the camelidæ a few of the comparatively large white globules, forming a singular contrast to the minute oval disks. The blood corpuscles of birds, though of the same shape as those we have been describing, are generally about twice the size, and differ also in structure. In the mammal, as has just been remarked, nothing like a central particle could be detected in the blood disk; but in birds, this may easily be demonstrated by various reagents; and it is very remarkable in its figure, being an extremely elongated ellipse. Thus, as is often the case, if the long diameter of the corpuscles of the bird be a little less than twice the short diameter, the nuclei will measure nearly thrice as much in length as in breadth.

From the vicugna and paco, I had an opportunity of obtaining some pus, and of ascertaining that it did not differ materially from that of animals with circular blood-disks. The globules of the pus of the former animal were extremely well defined, spherical, and rather less granular on the surface than common. They readily exhibited their central molecules when subjected to the action of sulphurous or acetic acids; with water the globules became enlarged, as I have often observed in the recent pus-globules of man and other animals. Their size when examined in their own serum, was generally from $\frac{1}{4000}$ th to $\frac{1}{3200}$ th of an inch; the extreme diameters being $\frac{1}{4800}$ th and $\frac{1}{2666}$ th. When treated 
with water they expanded to upwards of $\frac{1}{2000}$ th of an inch.* There were a few irregularly oval particles in the pus ; not more so, however, than may sometimes be seen in the pus of the horse or cat.

The pus of the paco had similar characters. The globules were remarkably clear, and presented very well defined outlines; their most frequent diameter was $\frac{1}{3200}$ th of an inch, and $\frac{1}{3000}$ th was also a common size ; many were only $\frac{1}{4570}$ th and $\frac{1}{4000}$ th, and several were as large as $\frac{1}{2668}$ th. There were, besides, some molecules varying from $\frac{1}{10000}$ th to $\frac{1}{8000}$ th of an inch in diameter. Under the action of acetic acid, the nuclei of the pus-globules, corresponding in size and appearance with the molecules, were im. mediately visible; and the entire globules were enlarged by the addition of water. Mixed with a little liquid ammonia, the pus became very ropy, and the globules soon disappeared. When some of the blood-corpuscles were seen with the pus-globules, the diameter of most of the latter was evidently greater than that of the former.

It should be observed that suppuration takes place often with difficulty, and generally very sparingly, in the camelidæ, as far as my experience goes. But though the pus is only obtained in small quantities, its characters, as previously described, are very well marked. The purulent matter in a very few days becomes tenacious and viscid, and indeed soon al-

* For some observations on this subject see Medical Gazette, Nov. 1st, 1839. 
most ceases to appear, a tough fibrinous mass occupying the wound.

Without asserting the impossibility of a transformation of the blood-disk into the globule of pus, it can hardly be supposed that any such change took place in the experiments recorded in this paper. This question, however, appears to me to be one of more difficult solution by mere microscopic observation than would be supposed by any one who had not specially examined the subject; for the blood-corpuscles are so singularly susceptible of modifications in form, size and general characters from very slight agency, that examples might readily be shown of their approximation in appearance to the globules of pus. The action of water on the blood-disks of the mammalia, as well as of the lower vertebrate animals, has been well known since the time of Hewson.

Now, however, that so much attention is devoted to the constitution of the healthy as well as morbid animal fluids, the relation, if any, between the blood corpuscles and the particles of the secretions will probably be soon finally determined. From my observations it appears that the blood-disks of the goat are by no means the smallest among the mammalia, as had been previously supposed, but that the bloodcorpuscles of the napu musk deer,* and probably of its congeners, are so singularly minute that their

* See London and Edin. Phil. Mag. for Dec. 1839, and Feb. 1840, p. 114. Dublin Med. Press, Nov. 1839. 
28 MR, GULLIVER ON BLOOD-CORPUSCLES, ETC.

average diameter is between $\frac{1}{14000}$ th and $\frac{1}{12000}$ th of an inch. It would, therefore, be interesting to examine the pus of an animal of this genus. In the meantime it may be mentioned that $I$ found in the blood of the musk deer several large white spherical bodies, similar to those observable in the blood of other animals, and that the lymph globules did not differ in magnitude from those of mammals with large blood-corpuscles. 